\title{
WAVES ON GLACIER BEDS
}

\author{
By V.L. Mazo
}

(Institute of Geography, U.S.S.R. Academy of Sciences, Moscow 109017, U.S.S.R.)

ABSTRACT. A joint dynamical system comprising both an eroding glacier and an eroded bed is considered to describe the formation of cirques and stepped longitudinal profiles of trough valleys. A glacier is modelled as a layer of a very viscous incompressible fluid flowing down an inclined bed. The rate of erosion is assumed to be determined by the basal shear stress. The dynamics of small longitudinal perturbations are studied. The analysis shows that the perturbations propagate up the glacier bed with velocities that are different for the perturbations of different spatial scales. As a result, a perturbation of a specific spatial scale stands out against the others and develops into the morphological forms considered here.

\section{INTRODUCTION}

Cirques and stepped longitudinal profiles of trough valleys are common features of glaciated, and only glaciated, mountain relief. Therefore, it is glacial erosion that causes these morphological forms. Davis wrote as far back as 1906 "that it savors of extreme conservatism any longer to deny the efficacy of glacial erosion". However, extreme conservatism is extremely conservative - after decades we have to demonstrate the statement again.

Modern estimates (Serebryannyy and Orlov, 1982; Gros'vald and Glazovskiy, 1983) suppose that the rate of glacial erosion is sufficiently high to create considerable morphological forms. But the next question arises: why glacial erosion is not homogeneous along the glacial bed, localizes in particular zones, and thus generates cirques and stepped longitudinal profiles within trough valleys. The main object of this paper is to demonstrate the reasons for self-organization of the longitudinal glacial trough profiles in principle. So some statements are only mentioned without detail.

According to widespread opinion, heterogeneities of glacial erosion occur because of a variety of different non-glacial and ad hoc reasons: pre-glacial bed configuration, lithological differences of bedrock, and others (see Colman's (1976) critical review). An alternative approach is to connect the heterogeneities of glacial erosion to heterogeneities of glacier flow. Clark and Lewis (1951) used the rotational flow of glaciers. Nye and Martin (1968) proposed the connection between the heterogeneities of glacial erosion and the active and passive flow of glaciers (Nye, 1952). However, both approaches require a particular pre-glacial relief to generate rotational or active and passive glacier flow, and therefore spatial scales of the erosional forms are prescribed in advance.

A glacier bed does not only vary under the impact of glacial erosion: glacier flow, in turn, varies according to changes in the bed. Therefore, we encounter a purely glaciological self-supported process for the generation of heterogeneities of glacier flow and bed configuration. It appears adequate to connect the dynamics of an eroding glacier and eroded bed to describe the genesis process. According to synergetic ideology (Haken, 1978), we should investigate the dynamics of small random perturbations against the background of a homogeneous glacier flow and a homogeneous glacier bed.
The approach proposed here has been used for transverse perturbations to explain the generation of ice-sheet ice streams and associated large-scale trough valleys (Mazo, 1987). In this case, the heterogeneities are due to the instability of the perturbations of larger scales and the stability of the perturbations of smaller scales, or due to negative or positive dissipation.

In this paper, the same approach is applied for longitudinal perturbations to investigate the formation of cirques and stepped longitudinal profiles within glacial troughs. The analysis below shows that, in contrast to the transverse perturbations, the longitudinal ones dampen out for all spatial scales: this attenuation is small for the perturbations of larger scales and is rapid for those of moderate and smaller scales. However, for the longitudinal perturbations, there exists another mechanism that creates the heterogeneities: the longitudinal perturbations propagate with non-zero velocities up the glacier bed and these velocities are different for the perturbations of different spatial scales. In other words, velocity dispersion arises. Moreover, for a certain spatial scale stationary phase (Whitham, 1974) occurs. The result is that the longitudinal perturbations of this spatial scale stand out against the others.

\section{GLACIER FLOW DOWN A PERTURBED BED}

A glacier is modelled here as a layer of a very viscous linear incompressible liquid flowing down an inclined bed by gravity. The following scales are chosen: spatial scale $d$, time-scale $d^{2} / v$, velocity scale $d^{2} g \sin \theta / 2 v$, pressure scale $\rho g d$, where $d$ is the average thickness of the layer, $\theta$ is the average angle of the bed slope, $\rho$ and $v$ are the density and the kinematic viscosity of the liquid, and $g$ is the acceleration of gravity. All equations are cast in terms of dimensionless variables. The $x$-axis is in the ice-flow direction, the $z$-axis is normal to the surface, positive upwards, $z=b(x)$ and $z=f(x)$ are the equations of the bed and free surface, $h(x)=f(x)-b(x)$ is the thickness of the layer (see Fig. 1).

The ice-layer flow is described by a system of the

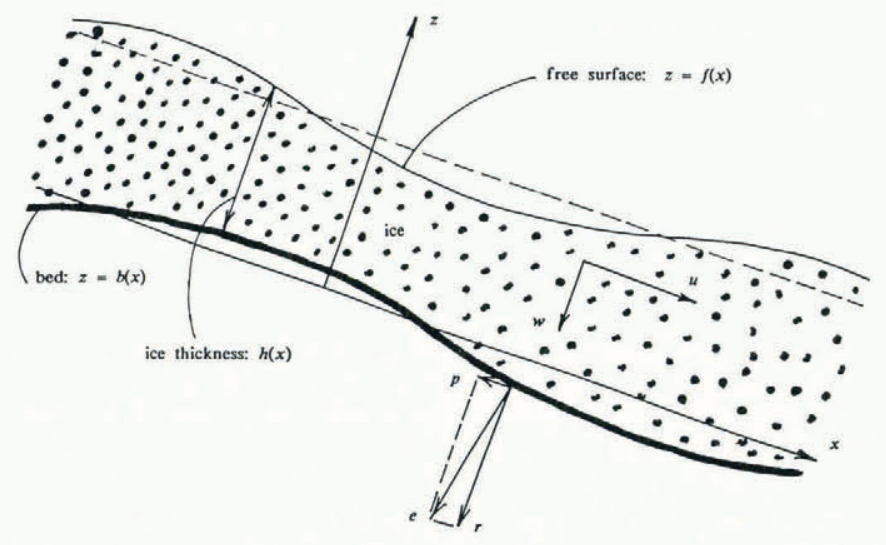

Fig. 1. Longitudinal section of a glacier. 
incompressibility and equilibrium equations and boundary conditions on the bed and free surface for the pressure and $x$ - and $z$-components of ice flow $u$ and $w$. The latter may be, in principle, found as a function of the boundaries $b$ and $f$. However, it is difficult because of the complexity of the full system and, more important, unnecessary because the short-scale perturbations dampen rapidly and, therefore, may be excluded from consideration. We will find the pressure, velocity components $u$ and $w$ and, in addition, the basal shear stress in long-wave approximation when the spatial scales of the perturbations are supposed to be much longer than the averaged ice thickness.

An additional boundary condition on the free surface is the kinematic equation

$$
f_{\mathrm{t}}+u f_{x}=w, \text { for } z=f(x),
$$

which in the long-wave approximation (see Appendix, Equation (A8)) is reduced to the non-linear parabolic equation of the free-surface relaxation

$$
f_{\mathrm{t}}+\sin \theta h^{2} h_{x}=(\cos \theta / 3)\left(h^{3} f_{x}\right)_{x}
$$

where $h=f-b$ and the bed is supposed to be given. This equation is a convenient tool to describe free-surface dynamics of thin layers for given beds. In particular, the relaxation equation describes propagation of free-surface waves which are shown to tend to the steady-state wave associated with the given bed wave.

The steady-state free-surface wave satisfies the stationary equation

$$
\sin \theta \underline{h}^{3}=\cos \theta \underline{h}^{3} f_{x}+\sin \theta
$$

where $\underline{h}=f-b$ (steady-state variables are indicated by underlined italics). The steady-state equation may be used to solve the inverse problem of reconstructing the bed configuration for the given free surface. However, only the long-wave perturbations may be reconstructed: in the longwave approximation, the inverse problem for short waves is incorrect.

In the case of small amplitudes (much smaller than the average ice thickness) of the bed and, hence, of free-surface perturbations, the relaxation and stationary equations are reduced. Let us represent the perturbed variables $f$ and $b$ as sums of mean variables and perturbations $\varphi$ and $\beta$. The relaxation equation for small perturbations is then the linear parabolic equation

$$
\varphi_{t}+\sin \theta(\varphi-\beta)_{x}=(\cos \theta / 3) \varphi_{x x} .
$$

The small free-surface perturbation, $\varphi$, relaxes to the small steady-state perturbation $\varphi$ satisfying the stationary equation

$$
\varphi_{x}=\delta(\varphi-\beta)
$$

where $\delta=3 \tan \theta$. There exist two solutions for Equation (2) which dampen out for large $x$, positive or negative

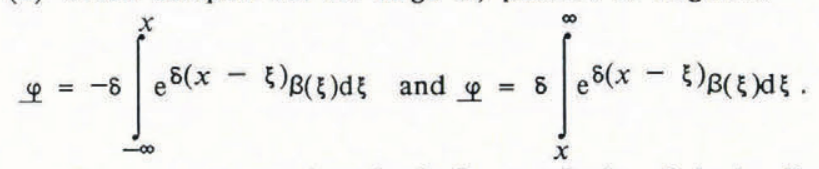

Let us suppose that the bed perturbation $\beta$ is localized within a section of the $x$-axis, and select a solution which decreases for large $x$, positive and negative. The first solution decreases for large negative $x$ while, generally, it increases for large positive $x$, and hence cannot be used. The second solution decreases in both the positive and negative directions and therefore can be used. To be more exact, the second solution is only non-zero up-stream of the local section where the solution decreases exponentially as $x \rightarrow \infty$, while it is zero everywhere down-stream of the section: bed perturbations result in free-surface perturbations that are only up-stream of the bed perturbations.

Let us proceed from the perturbations $\varphi$ and $\beta$ to their Fourier transforms $\varphi^{\prime}$ and $\beta^{\prime}$. We find from Equation (2)

$$
\varphi^{\prime}=\frac{1+i(k / \delta)}{1+(k / \delta)^{2}} \beta^{\prime}
$$

where $k$ is the wave number. Therefore, the ratio of the amplitudes of the stationary free-surface to the bed perturbations decreases as

$$
1 /\left[1+(k / \delta)^{2}\right]^{\frac{1}{2}}
$$

when the wave number $k$ increases: the longer the bed perturbations are, the less dampened will be the free-surface perturbations. More complex calculations show that the full system (not in the long-wave approximation) gives higher rates of the dampening of the short-wave perturbations than the system in the long-wave approximation: the short-wave perturbations indeed dampen out. Also, there is a phase lag between the perturbation (tangent of the phase-lag angle is $k / \delta$ ): long perturbations have a smaller lag.

\section{ERODED BED DYNAMICS}

In order to describe completely the dynamics of the joint system comprising both the eroding glacier and the eroded bed, an equation controlling the eroded bed changes must be added. It is the kinematic equation

$$
b_{t}+p b_{x}=r
$$

where $p$ and $r$ are $x$ - and $z$-components of the bed-erosion rate (see Fig. 1). The simplest assumption connecting the rate of bed erosion to the parameters of ice flow is as follows. The direction of the erosion-rate vector is supposed to be normal to the bed

$$
p=e\left[b_{x} /\left(1+b_{x}^{2}\right)^{\frac{1}{2}}\right], \quad r=-e\left[1 /\left(1+b_{x}^{2}\right)^{\frac{1}{2}}\right]
$$

where $e$ is the magnitude of erosion rate. The latter is supposed to be proportional to the basal shear stress $\tau_{\mathrm{b}}$ : $e=\varepsilon \tau_{\mathrm{b}}$, where $\varepsilon$ is a positive constant. Then the kinematic equation for erosion is

$$
b_{t}+\varepsilon \tau_{\mathrm{b}}\left(1+b_{x}^{2}\right)^{\frac{1}{2}}=0 .
$$

In the long-wave approximation, the equation reduces to

$$
b_{t}+\varepsilon \tau_{\mathrm{b}}=0 .
$$

Erosional changes of bed configuration occur on a time-scale $s$ which is much longer than the time-scale $t$ for the adjustment of the ice flow. Thus, at any given instant $s$ the bed configuration may be taken as the boundary condition for ice flow and the previous results for stationary ice flow may be used though the bed itself is being slowly eroded. It is the parameter $\varepsilon$ that characterizes the ratio of these time-scales: $s=\varepsilon t$. So, we may suppose that $\varepsilon \ll 1$.

Using the long-wave formula for the basal shear stress $\tau_{\mathrm{b}}$ (see Appendix, Equation (A9)), and proceeding from the shorter time-scale $t$ to the longer one $s$, we find the final form of the erosional kinematic equation

$$
b_{\mathrm{S}}+\sin \underline{\theta \underline{h}}=\cos \theta \underline{h f_{X}} \text {. }
$$

In the case of small amplitudes of the bed and, hence free-surface perturbations, the erosional kinematic equation is

$$
\beta_{S}=\mu(\varphi-\beta)
$$

where $\mu=2 \sin \theta$.

\section{PROPAGATION OF THE LONGITUDINAL BED WAVES}

Two Equations (1) and (2), or (3) and (4) (the latter in case of small-perturbation amplitudes), describe jointly the dynamics of the eroded bed and adjusted ice flow. Equations (3) and (4) are reduced to the single hyperbolic equation

$$
\beta_{\mathrm{s} x}-\delta \beta_{\mathrm{s}}+\mu \beta_{x}=0
$$

for which Equations (3) and (4) are characteristic. 
Let us proceed from the perturbation $\beta$ to its Fourier transform $\beta^{\prime}$. The latter varies exponentially with time: $B^{\prime}=$ constant $\exp (-a(k) s)$, where $a(k)$ is a wave-numberdependent complex frequency $(a(k)=\omega(k)+i \gamma(k), \omega(k)$ is a proper frequency, and $\gamma(k)$ is a time constant. The time constant $\gamma(k)$ determines the growth or dampening of the perturbation, while the phase velocity $c(k)=\omega(k) / k$ determines the propagation velocity of the perturbation. When $c(k)$ varies with $k$, velocity dispersion occurs. If, in addition to a velocity dispersion, the group velocity $g(k)=$ $\mathrm{d} \omega(k) / \mathrm{d} k$ is zero for certain wave numbers, then stationary phase occurs and the perturbations with these wave numbers stand out against the others.

From Equation (5) rewritten in terms of Fourier transforms, we find

$$
c(k)=-\frac{\mu / \delta}{1+(k / \delta)^{2}} ;
$$

so the phase velocity $c(k)$ is negative. Therefore, all longitudinal perturbations propagate up-stream. This circumstance is explained by the lag between the bed and free-surface perturbations (see previous discussion). Also, the phase velocity $c(k)$ is different for different wave numbers $k$ : thus the waves are dispersive. Moreover, the group velocity

$$
g(k)=-(\mu / \delta) \frac{1-(k / \delta)^{2}}{1+(k / \delta)^{2}}
$$

is zero for wave numbers $|k|=\delta$ and, therefore, for these critical wave numbers stationary phase occurs.

Thus, by the end of an initial transient period, the longitudinal bed wave with a wavelength which is $2 \pi / \delta \approx$ $2 / \tan \theta$ times longer than the ice thickness stands out against the others. This length is about the cirque-glacier length and several times shorter than the valley-glacier length: the longitudinal profile of a cirque corresponds to one period of the erosional wave and the longitudinal profile of a trough valley corresponds to several periods of the wave.

Additional calculations show that glacier-sliding conditions and/or non-linearity of ice rheology and the erosion law included in the model change the quantitative estimations slightly but do not change qualitative features of bed-wave propagation.

\section{ACKNOWLEDGEMENTS}

I thank G.K.C. Clarke and K. Hutter for their suggestions for improving both the presentation and my English.

\section{REFERENCES}

Benney, D.J. 1966. Long waves on liquid films. J. Math. Phys., 45(3), 150-155.

Clark, J.M. and W.V. Lewis. 1951. Rotational movement of cirque and valley glaciers. J. Geol., 59(6), 546-566.

Colman, S.M. 1976. Inherent factors in the flow of valley glaciers. Z. Geomorphol., 20(3), 297-307.

Davis, W.M. 1906. The sculpture of mountains by glaciers. Scot. Geogr. Mag., 22, 76-89.

Gros'vald, M.G. and A.F. Glazovskiy. 1983. Glacial erosion of continental margins. Geomorphologiya, 1, 3-14. [In Russian.]

Haken, H. 1978. Synergetics: an introduction. Nonequilibrium phase transformations and self-organization in physics. chemistry and biology. Second edition. Berlin, Springer Verlag.

Hutter, K. 1983. Theoretical glaciology; material science of ice and the mechanics of glaciers and ice sheets. Dordrecht, etc., D. Reidel Publishing Company.

Mazo, V.L. 1987. Effects of glacial erosion on the flow of ice sheets and the morphology of their beds. International Association of Hydrological Sciences Publication 170 (Symposium at Vancouver 1987 - The Physical Basis of Ice Sheet Modelling), 145-152.
Nye, J.F. 1952. The mechanics of glacier flow. J. Glaciol., 2(12), 82-93.

Nye, J.F. and P.C.S. Martin. 1968. Glacial erosion International Association of Scientific Hydrology Publication 79, (General Assembly of Bern 1967 - Snow and Ice), 78-86.

Serebryannyy, L.R. and A.V. Orlov, 1982. Glacier's impact on landscape in mountains. Geomorphologiya, 1, 22-32. [In Russian.]

Whitham, G.B. 1974. Linear and nonlinear waves. London, John Wiley and Sons.

\section{APPENDIX}

In the model considered, glacier flow is described by the equations of incompressibility and equilibrium

$$
\begin{aligned}
u_{x}+w_{z} & =0 \\
-p_{x}+\Delta u+\sin \theta & =0 \\
-p_{z}+\Delta w-\cos \theta & =0
\end{aligned}
$$

for the $x$ - and $z$-components of ice velocity $v$ and $w$, and the pressure $p$, where

$$
\Delta=\partial^{2} / \partial x^{2}+\partial^{2} / \partial y^{2}+\partial^{2} / \partial z^{2}
$$

is the Laplacian operator. The boundary conditions are: the no-slip conditions on the bed

$$
u=w=0, \text { for } z=b(x)
$$

and the zero-stress (normal and shear) conditions on the free surface

$$
\begin{aligned}
p+ & \frac{1-f_{x}^{2}}{1+f_{x}^{2}}\left(u_{x}-w_{z}\right)+\frac{2 f_{x}}{1+f_{x}^{2}}\left(u_{z}+w_{x}\right)=0, \text { for } z=f(x), \\
& \frac{1-f_{x}^{2}}{1+f_{x}^{2}}\left(u_{z}+w_{x}\right)-\frac{2 f_{x}}{1+f_{x}^{2}}\left(u_{x}-w_{z}\right)=0, \text { for } z=f(x) .
\end{aligned}
$$

The additional boundary condition on the free surface is the kinematic equation

$$
f_{t}+u f_{x}=w, \text { for } z=f(x) .
$$

Furthermore, the basal shear stress $\tau_{\mathrm{b}}$ is

$\tau_{\mathrm{b}}=\frac{1-b_{x}^{2}}{1+b_{x}^{2}}\left(u_{z}+w_{x}\right)-\frac{2 b_{x}}{1+b_{x}^{2}}\left(u_{x}-w_{z}\right)$, for $z=b(x)$.

The equation of incompressibility implies that the velocity components $u$ and $w$ may be deduced from the stream function $\psi: u=\psi_{z}, w=-\psi_{x}$. Let us express the velocity components $u$ and $w$ in terms of the stream function $\psi$ and eliminate the pressure $p$. The equations of equilibrium then reduce to the single equation

$$
\psi_{z z z z}+2 \psi_{z z x x}+\psi_{x x x x}=0
$$

and the kinematic Equation (A1) may be rewritten as the mass-conservation law

$$
f_{t}+q_{x}=0
$$

where $q=\psi_{z=f(x)}$ is the flux. The equation for basal shear stress $\tau_{\mathrm{b}}$ in terms of the stream function $\psi$ is 
$\tau_{\mathrm{b}}=\frac{1-b_{x}^{2}}{1+b_{x}^{2}}\left(\psi_{z z}+\psi_{x x}\right)-\frac{4 b_{x}}{1+b_{x}^{2}} \psi_{z x}$, for $z=b(x)$.

The problem is to find the stream function $\psi$ from Equation (A2) and the boundary conditions (except the kinematic Equation (A3)) and then substitute $\psi$ into the kinematic Equations (A3) and (A4) for the basal shear stress $\tau_{\mathrm{b}}$.

In the case of the small-amplitude perturbations, calculation shows that short-scale perturbations dampen rapidly and may be excluded from consideration. So we wil find the stream function $\psi$ in the long-wave approximation for which the spatial scales of the perturbations are supposed to be much longer than the average ice thickness. Here, we follow Benney (1966), whose approach is widely used in glaciology (see Hutter (1983) and papers quoted there). Equation (A2) is thus reduced to

$$
\psi_{z z z z}=0
$$

with the boundary conditions

$$
\begin{gathered}
\psi_{y}=\psi_{z}=0, \text { for } z=b(x), \\
\psi_{z z}=0, \text { for } z=f(x), \\
\psi_{z z z}=\cos \theta f_{x}-\sin \theta, \text { for } z=f(x) .
\end{gathered}
$$

The kinematic Equation (A3) remains the same. Equation (A4) for the basal shear stress $\tau_{\mathrm{b}}$ is reduced to

$$
\tau_{\mathrm{b}}=\psi_{z z}, \text { for } z=(x) \text {. }
$$

Solving the equilibrium Equation (A5), together with the boundary conditions (A6), we find

$$
q=\left.\psi\right|_{z=f(x)}=\left(h^{3} / 3\right)\left(\sin \theta-\cos \theta f_{X}\right),
$$

and hence we have for the kinematic Equation (A3)

$$
f_{t}+\sin \theta h^{2} h_{x}=(\cos \theta / 3)\left(h^{3} f_{x}\right)_{x} .
$$

For the basal shear-stress Equation (A7), we find

$$
\tau_{\mathrm{b}}=h\left(\sin \theta-\cos \theta f_{x}\right) .
$$

MS. received 2 August 1988 and in revised form 10 November 1988 